\title{
The role of radiological imaging for masses in the prevascular mediastinum in clinical practice
}

\author{
Helmut Prosch ${ }^{1}$, Sebastian Röhrich ${ }^{1}$, Zeynep Nilufer Tekin ${ }^{2}$, Lukas Ebner ${ }^{3}$ \\ ${ }^{1}$ Medical University of Vienna, Department of Biomedical Imaging and Image-guided Therapy, Vienna, Austria; ${ }^{2}$ Medeniyet University Goztepe \\ Training and Research Hospital, Department of Radiology, Istanbul, Turkey; ${ }^{3}$ Department of Diagnostic, Interventional and Pediatric Radiology, \\ Inselspital, Bern University Hospital, University of Bern, Bern, Switzerland \\ Contributions: (I) Conception and design: H Prosch, L Ebner; (II) Administrative support: S Röhrich, ZN Tekin; (III) Provision of study materials or \\ patients: All authors; (IV) Collection and assembly of data: All authors; (V) Data analysis and interpretation: All authors; (VI) Manuscript writing: All \\ authors; (VII) Final approval of manuscript: All authors. \\ Correspondence to: Assoc. Prof. Helmut Prosch, MD. Medical University of Vienna, Department of Biomedical Imaging and Image-guided Therapy, \\ Vienna General Hospital, Währingergürtel 18-20, 1090 Vienna, Austria. Email: helmut.prosch@meduniwien.ac.at.
}

\begin{abstract}
Tumors in the prevascular compartment of the mediastinum are rare and imaging plays a major role in their detection, (differential) diagnosis, staging, and follow-up. The prevascular compartment is bordered anteriorly by the posterior aspect of the sternum, posteriorly by the ventral aspect of the pericardium, cranially by the thoracic outlet, and caudally by the diaphragm. In many cases, the diagnosis of a lesion in the prevascular compartment is an incidental finding either on chest radiograph (CR) or on computed tomography (CT) scans. The differential diagnosis of masses in the pre-vascular mediastinum include primarily tumors arising from the thymus or the thyroid gland, lymphomas and germ cell tumors. The differential diagnosis of mediastinal masses is primarily based on the location of the mass, its tissue composition (i.e., fat content, calcifications) and the age of the patient. The imaging method of choice is CT, as it combines a high spatial and temporal resolution with the ability to determine tissue composition and detect fluid components, as well as areas of fat and calcifications. MRI is used as a more specific problemsolving tool to discriminate solid lesions from cystic lesions or to provide evidence of minimal fat content in teratoma and thymic rebound. The role of PET/CT in the evaluation of tumors other than lymphomas in the prevascular compartment is still under discussion.
\end{abstract}

Keywords: Mediastinal tumors; thymoma; mediastinal teratoma; mediastinal lymphoma

Submitted Feb 21, 2020. Accepted for publication Jun 15, 2020.

doi: $10.21037 /$ jtd-20-964

View this article at: http://dx.doi.org/10.21037/jtd-20-964

\section{Introduction}

Tumors in the prevascular compartment of the mediastinum are rare and imaging plays a major role in their detection, (differential) diagnosis, staging, and follow-up. The prevascular compartment is bordered anteriorly by the posterior aspect of the sternum, posteriorly by the ventral aspect of the pericardium, cranially by the thoracic outlet, and caudally by the diaphragm (1).

The differential diagnosis of mediastinal masses, in general, is primarily based on the location of the mass, its tissue composition (i.e., fat content, calcifications) and the age of the patient. The differential diagnosis of masses in the pre-vascular mediastinum are commonly referred to as the "terrible Ts" (Table 1) (2).

\section{Imaging of lesions in the prevascular compartment}

In many cases, the diagnosis of a lesion in the prevascular compartment is an incidental finding either on chest radiograph (CR) or on computed tomography (CT) 
Table 1 Differential diagnosis of masses in the pre-vascular mediastinum (2)

Thymic masses
Thymic hyperplasia
Thymic rebound
Thymoma
Thymic cancer
Thymic cysts
Thyroid masses
Thyroid goiter
Thyroid cancer
Terrible lymphomas
Teratomas (Germ cell tumors)

scans. For thymomas, few patients present with symptoms like myasthenia (and only about $15 \%$ of patients with myasthenia actually have a thymoma). For thymic neoplasms in general, symptoms may be caused by local extension and compression of neighboring structures.

\section{CT}

In cases where the suspicion of a mediastinal lesion is raised on CR, further imaging work-up is warranted. The imaging modality of choice to further evaluate masses in the prevascular compartment is contrast-enhanced CT, as it enables the visualization of mediastinal masses with a highspatial and temporal resolution. Furthermore, CT allows for the characterization of tissue components in mediastinal masses, which has a major impact on the differential diagnosis of mediastinal masses. In particular, the proof of fat within a heterogeneous mass in the prevascular compartment is critical, as this is virtually diagnostic for a benign teratoma (Figure 1) (1). In addition, calcifications or cystic components are of diagnostic value.

The role of CT in the evaluation of masses in the prevascular compartment was shown in a study among 127 patients with histologically verified masses of the prevascular mediastinum (3). In this study, the first-choice CT diagnosis was correct in $61 \%$ of the cases, whereas the first-choice MR diagnosis was correct in only $56 \%$ (3).

In addition to its role in the differential diagnosis, CT is also an indispensable tool for the staging of masses in the prevascular mediastinum, as it allows a determination of local invasion and lymph node status.

\section{Magnetic resonance imaging (MRI)}

While CT is the imaging method of choice for the initial evaluation of masses in the prevascular mediastinum, MRI is used as a problem-solving tool to further investigate known masses. MRI has proven to be a valuable tool in the differentiation of normal thymus, thymic hyperplasia, and neoplastic lesions, as well as vascular invasion. MRI is particularly helpful for the diagnosis and characterization of cystic lesions (Figure 2) and for discerning thymic hyperplasia from other thymic masses (Figure 3) (4). Whereas thymic hyperplasia shows a decrease in signal intensity in chemical-shift MRI, other thymic tumors do not show this decrease in signal.

\section{Positron emission tomography (PET)}

The role of nuclear medicine and particularly PET using fluorodeoxyglucose (FDG) as a tracer is not well established. Although higher FDG uptake values indicate malignancy, biopsy confirmation is also still required, as masses with low FDG uptake may be malignant and masses with a high FDG uptake can be benign (1). Consequently, FDG-PET/CT is primarily used for lymphomas, in lymph node staging, and for the detection of distant metastases.

\section{Differential diagnosis of masses in the pre- vascular mediastinum}

\section{Thymic masses}

\section{Normal thymus}

Generally, the thymus appears as a soft tissue structure in the anterior mediastinum. In newborns, the gland defines the contour of the mediastinum on plain radiographs. During puberty, the normal thymus gland continues to grow, reaching its peak weight at approximately 19 years of age. Subsequently, the thymus shrinks in size and undergoes lipomatous degeneration. By 40 to 60 years of age, the gland is mostly replaced by fatty tissue. On imaging studies, the thymus appears as a triangular-shaped structure with variable fat content. The edges of the normal thymus are straight or even concave. The size may vary according to age, as outlined above; several authors have also described a considerable variation with inspiratory levels (5). 


\section{Thymic cyst}

Thymic cysts may be congenital or acquired, and have an unilocular or multilocular appearance with a thin wall. The CT density of the liquid content ranges between $\sim 20$ to 60 Hounsfield units, depending on the presence of associated hemorrhage or infection. MRI greatly facilitates the diagnosis of cysts by showing fluid-like signal in T2-

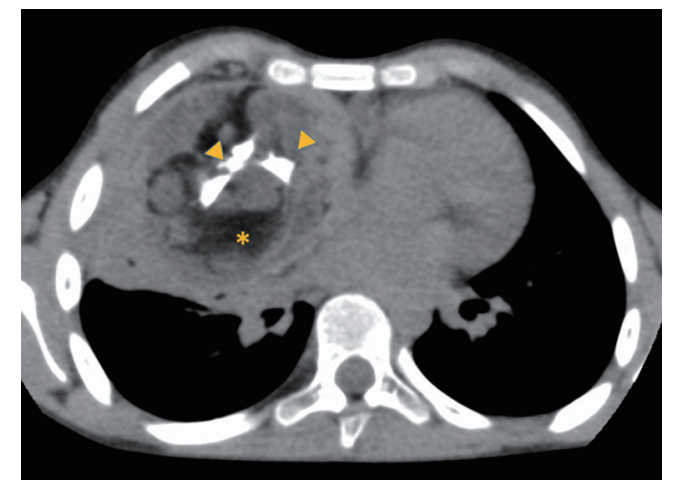

Figure 1 A 9-year-old female patient with increasing shortness of breath and chest pain. CT revealed an extensive mass in the prevascular compartment with areas of fat (asterix) and coarse calcification (arrowheads). Histology of the resected tumor confirmed the suspicion of a mature teratoma. weighted sequences (6). Differential diagnoses of thymic cysts are cystic thymomas or germ cell tumors, which show contrast-enhancing septa, whereas thymic cysts do not present with internal enhancement (6).

\section{Thymic byperplasia}

Thymic hyperplasia or rebound may occur at any age due to exogenous stressors and/or illness. On CT, thymic hyperplasia is characterized by new growth of soft tissue in the anterior mediastinum beyond the mentioned age characteristics. In addition, bulky and convex borders indicate growth. Whereas the patient's history may suggest thymic rebound, with CT alone, no formal differentiation from malignant lesions is feasible. However, MR imaging has proven to be a valuable method by which to discern normal thymus and hyperplasia from thymic neoplasm. Ackman et al. were able to show that chemical-shift imaging could reliably differentiate between benign hyperplasia and malignant tumors. On in- and out-of-phase gradient-echo (GRE) imaging, thymus and thymic hyperplasia show a decrease in signal intensity on out-of-phase images because of the presence of microscopic fat (Figure 3). In contrast, thymic neoplasms do not exhibit decreasing signal intensity on opposed-phase GRE imaging (7).
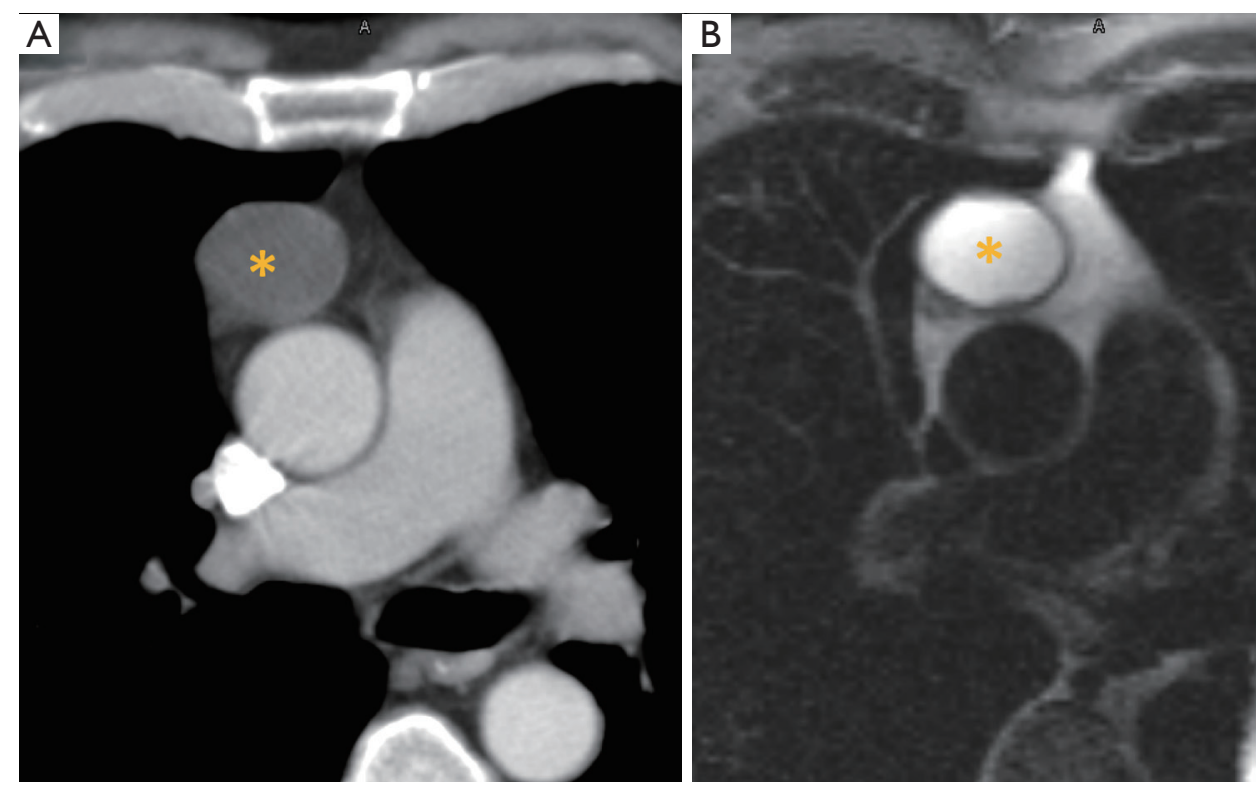

Figure 2 A 64-year-old male patient with an incidental finding of a soft tissue mass in the prevascular mediastinum. (A) CT shows a homogenous, well defined oval shaped mass (asterix). (B) On the corresponding MRI the mass (asterix) appears as thin walled lesion with a homogenous hyperintense content, corresponding to a thymic cyst. 

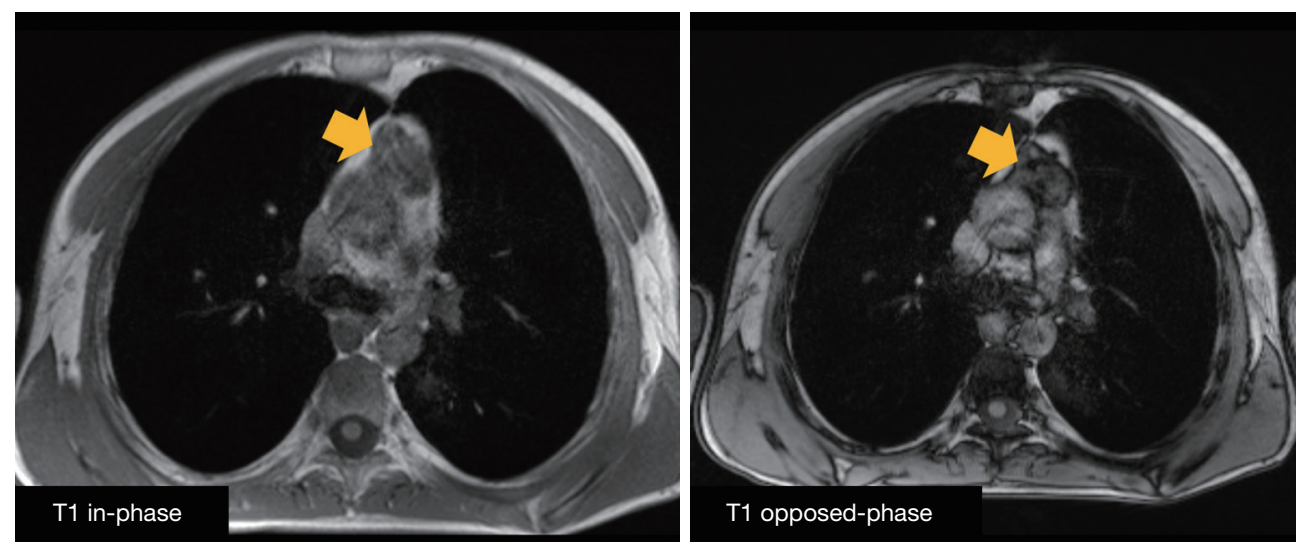

Figure 3 MRI of a 33-year-old male patient four years after lung transplantation. On the T1-weighted sequence, there is an oval-shaped mass in the prevascular compartment (arrows), which shows a drop in signal on the T1 opposed-phase sequence, confirming the suspicion of a thymic hyperplasia (thymic rebound).

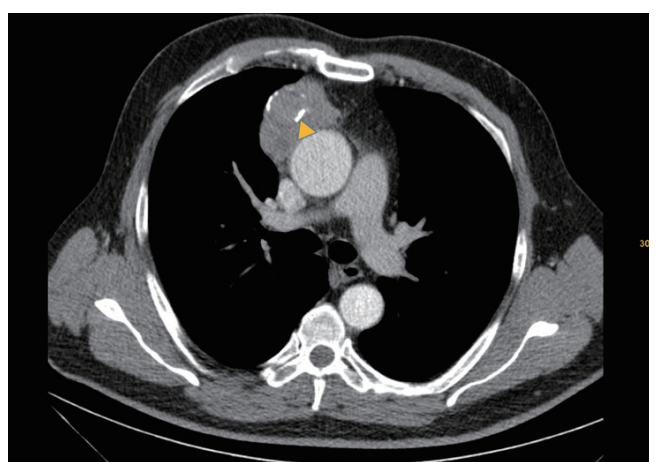

Figure 4 A 51-year-old male patient with an incidental finding of a mass in the anterior mediastinum. CT shows a soft-tissue mass in the anterior mediastinum with coarse calcifications (arrowhead). Biopsy revealed a thymoma.

\section{Thymic epithelial malignancies}

Thymic epithelial tumors are truly rare entities, with an incidence between 0.3 to 1.5 per million per year. Epithelial tumors are stratified into thymoma (Figure 4), thymic carcinoma (Figure 5), and neuroendocrine tumors of the thymus (4). On imaging, a differentiation of these entities is not reliably feasible.

As outlined above, CT is mainly applied for staging in these patients. Thymic malignancies are staged according to the TNM staging system (UICC $8^{\text {th }}$ edition) released in 2017 (8). The TNM staging of thymic epithelial tumors is briefly summarized in Table 2 .

Contrast-enhanced CT is capable of depicting local infiltration of the lungs, the brachiocephalic vein and the vena cava, the pulmonary arteries, and the aorta, as well as the trachea and esophagus, thus helping to separate the different tumor stages (9). Furthermore, macroscopic pleural invasion can be detected. Infiltration is suspected if either direct invasion is seen, or, if there is a broad contact between the lesion and other structures. With increasing contact area, infiltration becomes more likely. However, the assessment of a peri- and/or myocardial invasion can be challenging at times because of limited soft tissue resolution. Additional MR imaging can be helpful in the evaluation of cardiac and vessel involvement.

Local lymph node metastasis can be assessed with either CT or PET/CT. In cases where imaging reveals equivocal results, tissue sampling might be required.

\section{Thyroid masses}

\section{Thyroid goiter}

Thyroid goiters account for about $10 \%$ of all mediastinal masses and are, in most cases ( $75 \%$ to $90 \%$ of all cases), located in the prevascular compartment (10). Due to their expansive growth, thyroid goiters can lead to a displacement or narrowing of the trachea. The radiological diagnosis of thyroid goiters is usually straightforward, as they appear hyperdense on non-enhanced CT scans (>100 HU) and show a prolonged enhancement on contrast-enhanced scans (10). Calcifications are commonly seen.

\section{Thyroid cancers}

Mediastinal thyroid cancers should be suspected in illdefined thyroid masses or in case of regional lymph node 


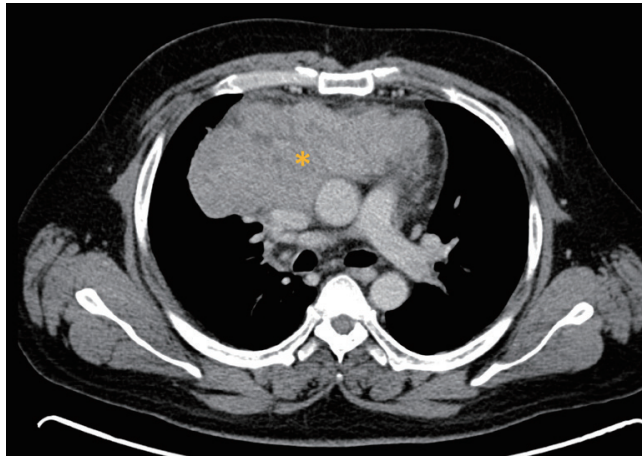

Figure 5 A 39-year-old female patient with increasing fatigue and shortness of breath. The CT scan shows a mostly homogenous, partly ill-defined mass in the prevascular compartment. Biopsy revealed a squamous cell carcinoma of the thymus (asterix).

enlargement.

\section{(Terrible) Lymphoma}

Lymphomas account for up to $20 \%$ of mediastinal tumors in adults. The mean age at diagnosis is 30 years (11). Mediastinal tumors are usually seen as part of a thymic involvement of a systemic disease. About $50 \%$ to $70 \%$ of mediastinal lymphomas are Hodgkin's lymphomas (11).

On CT, mediastinal lymphomas typically present as a large homogenous lobulated or nodular mass in the prevascular compartment that may encircle vascular structures, but does not infiltrate them (Figure 6) (1). The latter feature is helpful in discriminating mediastinal lymphomas from malignant epithelial tumors, which tend to infiltrate neighboring structures. Prior to treatment, mediastinal lymphomas usually do not appear calcified, which is also an important aspect to be considered in the differential diagnosis. Furthermore, some mediastinal lymphomas may show areas of tissue necrosis.

While the use of FDG PET/CT in epithelial tumors of the thymus is debatable, FDG PET/CT is a cornerstone in the staging of mediastinal lymphomas. As patients with mediastinal lymphomas are typically rather young, MRI with the use of diffusion-weighted images is an attractive alternative to PET/CT in this scenario (12).

\section{Mediastinal germ cell tumors}

Germ cell tumors originate from primitive germ cell layers, which may be found dispersed in the anterior mediastinum as
Table 2 TNM staging of thymic epithelial tumors (8)

\begin{tabular}{ll}
\hline Descriptor & \multicolumn{1}{c}{ Definition } \\
\hline T descriptor & \\
T1a & $\begin{array}{l}\text { Encapsulated or unencapsulated, with or } \\
\text { without extension into mediastinal fat }\end{array}$ \\
T1b & Extension into mediastinal pleura \\
T2 & Involvement of pericardium \\
T3 & $\begin{array}{l}\text { Involvement of lung, brachiocephalic vein, } \\
\text { superior vena cava, chest wall, phrenic } \\
\text { nerve, hilar (extrapericardial) pulmonary } \\
\text { vessels } \\
\text { T4 }\end{array}$ \\
Involvement of aorta, arch vessels, main \\
pulmonary artery, \\
myocardium, trachea, or esophagus \\
N descriptor & No lymph node involvement \\
N0 & Anterior (perithymic) lymph nodes \\
N1 & Deep intrathoracic or cervical lymph nodes \\
N2 & Pulmonary intraparenchymal nodule or \\
M descriptor & distant organ metastasis \\
M1a & \\
M1b &
\end{tabular}

a result of aberrant migration of primordial germ cells (11). In adults, germ cell tumors account for up to $15 \%$ of all mediastinal tumors (11). Germ cell tumors are subdivided into germinomatous and non-germinomatous germ cell tumors. Germinomatous germ cell tumors include seminomas and germinomas. Non-germinomatous tumors include all other germ cell tumors. The most common germ cell tumors are teratomas, which frequently present with distinct imaging features. Most are mature (benign) and, on CT, they commonly present as a well-defined mass in the anterior mediastinum with a variable extent of cystic degeneration, calcification, and areas of fatty tissue (10). Suggestive for malignant teratomas are focal contrastenhancing areas or signs of an infiltration of neighboring structures (10).

Seminomas, the second most common type of germ cell tumors, present as a homogenous mass in the anterior mediastinum, and consequently, share imaging features with mediastinal lymphomas (4). Elevated serum $\beta$-HCG levels associated with normal alpha-fetoprotein levels may help with the diagnosis, which can be observed in up to $10 \%$ of 


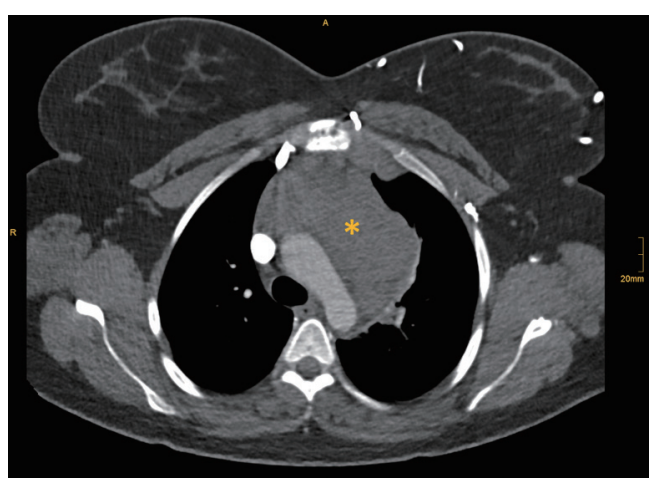

Figure 6 A 25 -year-old female patient with chest pain. CT revealed an extensive, well-defined homogenous mass, which proved to be a Hodgkin's lymphoma (asterix).

patients with mediastinal seminomas (4).

\section{Conclusions}

Imaging plays a pivotal role in the evaluation of masses in the prevascular compartment. CT is the imaging method of choice to investigate patients with such masses, as it has an excellent spatial and temporal resolution. While certain masses, such as goiter and teratomas, have pathognomonic CT features, others require further evaluation using MRI or a biopsy.

\section{Acknowledgments}

Funding: This work was supported by an unrestricted grant from Stiftung Oskar Helene Heim, Berlin, Germany.

\section{Footnote}

Provenance and Peer Review: This article was commissioned by the Guest Editors (Dragana Jovanovic and Semra Bilaceroglu) for the series "Thymoma" published in fournal of Thoracic Disease. The article was sent for external peer review organized by the Guest Editors and the editorial office.

Conflicts of Interest: All authors have completed the ICMJE uniform disclosure form (available at: http://dx.doi. org/10.21037/jtd-20-964). The series "Thymoma” was commissioned by the editorial office without any funding or sponsorship. HP reports grants and personal fees from Boehringer Ingelheim, personal fees from Roche, personal fees from MSD, personal fees from BMS, personal fees from AstraZeneca, personal fees from Siemens, outside the submitted work. The other authors have no conflicts of interest to declare.

Ethical Statement: The authors are accountable for all aspects of the work in ensuring that questions related to the accuracy or integrity of any part of the work are appropriately investigated and resolved.

Open Access Statement: This is an Open Access article distributed in accordance with the Creative Commons Attribution-NonCommercial-NoDerivs 4.0 International License (CC BY-NC-ND 4.0), which permits the noncommercial replication and distribution of the article with the strict proviso that no changes or edits are made and the original work is properly cited (including links to both the formal publication through the relevant DOI and the license). See: https://creativecommons.org/licenses/by-nc-nd/4.0/.

\section{References}

1. Carter BW, Benveniste MF, Madan R, et al. ITMIG Classification of Mediastinal Compartments and Multidisciplinary Approach to Mediastinal Masses. Radiographics 2017;37:413-36.

2. Quint LE. Imaging of anterior mediastinal masses. Cancer Imaging 2007; 7 Spec No A:S56-62.

3. Tomiyama N, Honda O, Tsubamoto M, et al. Anterior mediastinal tumors: diagnostic accuracy of CT and MRI. Eur J Radiol 2009;69:280-8.

4. Carter BW, Okumura M, Detterbeck FC, et al. Approaching the patient with an anterior mediastinal mass: a guide for radiologists. J Thorac Oncol 2014;9:S110-8.

5. Nasseri F, Eftekhari F. Clinical and Radiologic Review of the Normal and Abnormal Thymus: Pearls and Pitfalls. Radiographics 2010;30:413-28.

6. Ackman JB, Verzosa S, Kovach AE, et al. High rate of unnecessary thymectomy and its cause. Can computed tomography distinguish thymoma, lymphoma, thymic hyperplasia, and thymic cysts? Eur J Radiol 2015;84:524-33.

7. Ackman JB, Kovacina B, Carter BW, et al. Sex Difference in Normal Thymic Appearance in Adults 20-30 Years of Age. Radiology 2013;268:245-53.

8. Detterbeck FC, Stratton K, Giroux D, et al. The IASLC/ ITMIG Thymic Epithelial Tumors Staging Project: proposal for an evidence-based stage classification system for the forthcoming (8th) edition of the TNM classification 
of malignant tumors. J Thorac Oncol 2014;9:S65-72.

9. Marom EM, Milito MA, Moran CA, et al. Computed tomography findings predicting invasiveness of thymoma. J Thorac Oncol 2011;6:1274-81.

10. Thacker PG, Mahani MG, Heider A, et al. Imaging Evaluation of Mediastinal Masses in Children and Adults: Practical Diagnostic Approach Based on A New
Classification System. J Thorac Imaging 2015;30:247-67.

11. Shahrzad M, Le TS, Silva M, et al. Anterior mediastinal masses. AJR Am J Roentgenol 2014;203:W128-38.

12. Mayerhoefer ME, Karanikas G, Kletter K, et al. Evaluation of diffusion-weighted MRI for pretherapeutic assessment and staging of lymphoma: results of a prospective study in 140 patients. Clin Cancer Res 2014;20:2984-93.

Cite this article as: Prosch H, Röhrich S, Tekin ZN, Ebner L. The role of radiological imaging for masses in the prevascular mediastinum in clinical practice. J Thorac Dis 2020;12(12):75917597. doi: $10.21037 /$ jtd-20-964 